\title{
DESCRIPCIÓN DE LOS NIDOS DEL COCODRILO DE PANTANO CROCODYLUS MORELETII EN UN PAISAJE URBANIZADO EN EL SURESTE DE MÉXICO
}

\author{
Marco A. López-Luna, ${ }^{1}$ Mircea G. Hidalgo-MiharT ${ }^{1}$ \& Gustavo $^{-}$ \\ AgUiRre-León ${ }^{2}$
}

${ }^{1}$ División Académica de Ciencias Biológicas, Universidad Juárez Autónoma de Tabasco. Carr. Villahermosa-Cárdenas km. $0.5 \mathrm{~s} / \mathrm{n}$ Entrq. Bosques de Saloya 86039, Villahermosa Tabasco, MÉXICO ${ }^{2}$ Instituto de Ecología, A.C. Red de Interacciones Multitróficas. Carretera antigua a Coatepec No. 351, El Haya 91070, Xalapa, Veracruz, MÉXICO

e-mail: marco.lopez@dacbiol.ujat.mx

López-Luna, M. A., M. G. Hidalgo-Mihart \& G. Aguirre-León. 2011. Descripción de los nidos del Cocodrilo de pantano Crocodylus moreletii en un paisaje urbanizado en el sureste de México. Acta Zool. Mex. (n. s.), 27(1): 1-16.

RESUMEN. La Laguna de las Ilusiones es un lago urbano aislado en la ciudad de Villahermosa, Tabasco que mantiene una población de cocodrilo de pantano (Crocodylus moreletii). Durante los años 2007 y 2008 se estudiaron 22 nidos silvestres de esta especie. En la mayoría de los nidos (53\%) además de estar construidos con material vegetal (hojas, tallos y ramas delgadas) se registran productos de desecho humano como parte de los materiales para construcción de los nidos, el resto de los nidos está construido como lo descrito en otros trabajos. El tamaño promedio de la nidada es de $30.4 \pm 10.8$ huevos por nido (nidos $n=19$, huevos $n=578$ ). El tamaño y volumen promedio de los huevos es significativamente diferente entre nidos, lo que indica que existen diferencias en los tamaños de las hembras nidificantes. Las características físicas de los nidos en 2007-2008 fueron comparadas con las de otros nidos encontrados en la misma área de estudio en el periodo 1991-1994, encontrando que el diámetro de los nidos, la distancia a la orilla y el tamaño de la nidada son significativamente mayores en 2007-2008. El porcentaje de eclosión en 2007-2008 en la Laguna de las Ilusiones es bajo (33.9 \pm 31.21$)$ comparado con otras especies de cocodrilianos. El 100\% de los neonatos en 2007 y 2008 fueron machos. El efecto de la ciudad posiblemente puede afectar la ecología de la anidación en el cocodrilo de pantano, influyendo en nuevos materiales para la construcción de los nidos, aumentando el tamaño del área basal de los nidos y promoviendo la búsqueda de lugares más alejados de la orilla para la anidación. Debido al fuerte sesgo que existe hacia los machos en los nacimientos, se recomienda un manejo de la de la población de cocodrilos que incluya la reintroducción de hembras e incubación artificial en la Laguna de las Ilusiones que coadyuve a la conservación de esta población en esa área.

Palabras clave: éxito de eclosión, hábitat de anidación, tamaño de nidada, tamaño de huevo, proporción de sexos de neonatos.

Recibido: 11/08/2009; aceptado: 01/10/2010. 
López-Luna, M. A., M. G. Hidalgo-Mihart \& G. Aguirre-León. 2011. Description of Morelet's crocodile Crocodylus moreletii nests from an urban landscape in southeastern Mexico. Acta Zool. Mex. (n. s.), 27(1): 1-16.

ABSTRACT. "Laguna de las Ilusiones" is an isolated urban lake in the city of Villahermosa, Tabasco in México that retains a population of Morelet's crocodile (Crocodylus moreletii). During the years 2007 and 2008 we studied 22 natural nests of this species. Most of the nests $(53 \%)$ were built with vegetal material (leaf-litter, stems and thin branches). Also, we recorded human waste as part of the materials for nest construction. Mean clutch size was $30.4 \pm 10.8$ eggs by nest (nests $n=19$, eggs $n=578$ ). Mean egg volume is significantly different among nests, an indication of size differences between the nesting females. Physical characteristics of the nests in 2007-2008 were compared with nests found at the same area during 1991-1994. We found that nest diameter, shore distance and clutch size were significantly higher in 2007-2008. Hatching in "Laguna de las Ilusiones" was low during 2007-2008 (33.9\% \pm 31.21$)$ compared with other crocodilians. All new born crocodiles in 2007 and 2008 were males. We discuss how the city may affect the nesting of Morelet's crocodiles, influencing different aspects such as, the use of new materials for nest construction, increasing the size of the basal area of the nests, or promoting the search for a nesting site away from the shore. We recommend a management of the crocodiles with female reintroduction and artificial incubation in the "Laguna de las Ilusiones" to facilitate conservation of this population.

Key words: clutch size, egg size, hatching success, neonate sex ratio, nesting habitat.

\section{INTRODUCCIÓN}

Crocodylus moreletii es conocido en México con los nombres comunes de lagarto o cocodrilo negro, pardo o de pantano, se encuentra dentro de la lista de especies bajo la categoría de Bajo protección Especial en la NOM-059-ECOL-2001 (SEMARNAT 2002), en el Apéndice I de CITES y según la UICN/CSG con estatus de riesgo menor dependiente de la conservación (Ross 2000). El cocodrilo de pantano se puede encontrar en la costa del Golfo de México, desde el centro y posiblemente norte de Tamaulipas hasta la península de Yucatán, Belice y norte de Guatemala. En México se distribuye en los estados de Campeche, Chiapas, Oaxaca, Quintana Roo, San Luis Potosí, Tabasco, Tamaulipas, Veracruz y Yucatán, en las zonas bajas de la planicie costera hasta una altitud de $350 \mathrm{msnm}$ (Domínguez-Laso 2006). Es un habitante de agua dulces como arroyos, ciénagas, lagunas que están dentro de bosques y selvas, en ríos de corriente lenta y muy raras veces en ríos caudalosos y es común encontrarlo tanto en aguas claras como turbias, con abundante vegetación (Platt \& Thorbnarjarson 2000). En los últimos años el cocodrilo de pantano ha sido una especie muy utilizada para la crianza intensiva por su facilidad de adaptación al cautiverio y en México ha sido introducido en algunas localidades fuera de su rango natural de distribución, como en los estados de Sinaloa y Colima con fines comerciales (INE 1999). Mucha información sobre su reproducción y comportamiento es anecdótica y las observaciones sobre la biología en vida silvestre son escasas y dispersas. Se sabe que el cocodrilo de pantano es la única especie de Crocodylus del nuevo mundo que construye exclusivamente montículos para la anidación (Campbell 1972, Thorbjarnarson 1996) y se han descrito eventos sobre la reproducción como comportamiento 
maternal (Hunt 1973, 1975, 1977), o tamaños de camada (Casas-Andreu \& RogelBahena 1986). En México la mayoría de los trabajos han sido observaciones en cautiverio (Casas-Andreu \& Guzmán-Arroyo 1970, Álvarez del Toro 1974, Sigler \& González-Blanco 1994) y muy pocas en vida silvestre (Pérez Higareda 1980, Sigler \& Marina 2004). Recientemente han sido publicados estudios sistemáticos sobre el estatus de sus poblaciones (Platt \& Thorbjarnarson 2000) y su reproducción en Belice, donde se describen eventos de la reproducción en vida silvestre como periodo de anidación, características de los nidos, tamaños de camada y tallas a la madurez entre otros parámetros (Platt et al. 2008).

La modificación, pérdida y degradación de los humedales para el uso y aprovechamiento humano ha sido un factor muy importante que impacta las poblaciones de cocodrilianos (Ross 1998), muchas de estas poblaciones se encuentran al borde de la extinción (p. ej. Alligator sinensis en China, Thorbjarnarson et al. 2002), o bien, demuestran cierta plasticidad que les permite permanecer en ambientes transformados con resultados variables (p. ej. Caiman latirostris, Borteiro et al. 2008, Verdade 1998). Los ambientes urbanos pueden tener efectos sobre la biología de muchas especies, modificando aspectos de la historia de vida, nutrición, reproducción, demografía, etc. (Faeth et al. 2003, Shochat et al. 2006). Estos efectos pueden reflejarse de manera inmediata (como en el caso de la construcción de grandes obras de infraestructura) o bien a largo plazo por modificaciones graduales (Grimm et al. 2008). En este último caso, las ciudades en crecimiento con cuerpos de agua en el interior pueden encerrar a poblaciones de animales silvestres con hábitos acuáticos. Entender como el cocodrilo de pantano utiliza el hábitat puede ser muy útil para la elaboración de estrategias para la conservación y manejo de las poblaciones de esta especie en el interior de las ciudades (Bodie 2001, Joyal et al. 2001). El conocimiento sobre la existencia de poblaciones de cocodrilos en ambientes urbanos (Domínguez-Laso 2006) y los estudios poblacionales de cocodrilianos en estos ambientes antrópicos son escasos (Borteiro et al. 2008). Existe muy poca información publicada sobre la anidación de cocodrilos en ambientes urbanos (e. g., Gómez-González 1995, Pacheco de la Cruz 1996), por lo que el conocimiento generado en este tipo de paisajes permite obtener información novedosa y útil para la conservación de los cocodrilos en ambientes atípicos.

En la población cocodrilos de pantano que habitan el ambiente urbano de Villahermosa, Tabasco, se describió y analizó en este trabajo en: 1) la anidación (tamaño del nido, materiales de construcción del nido, distancia del nido a la orilla), 2) parámetros de la puesta (número de huevos, tamaño de los huevos y proporción sexual de las crías) y 3) tamaño de las hembras que anidan en el área de estudio.

\section{MATERIAL Y MÉTODOS}

Los datos de los nidos de cocodrilo de pantano fueron obtenidos durante los años de 2007 y 2008 en el lago natural urbano "Laguna de las Ilusiones" (1800’41" N 
$92^{\circ} 55^{\prime} 55^{\prime \prime}$ W Figura 1), el cual tiene una superficie de 260 ha y se encuentra aislado dentro la ciudad de Villahermosa, capital del estado de Tabasco en México. Su clima es Am(f), según la clasificación de Köppen modificada por García (1988), y se considera cálido húmedo con lluvia abundante todo el año y un pico en la precipitación en verano, la temperatura media anual es de $27^{\circ} \mathrm{C}$. La precipitación del mes más seco es de $47.7 \mathrm{~mm}$ y el porcentaje de lluvia invernal respecto al total anual es de $18 \%$ (Pereyra et al. 2003). Muy alterada por la urbanización, la vegetación que rodea la laguna cubre la mayor parte de la orilla y se extiende por lo general a unos cuantos metros de ésta (Magaña 1988). Entre las especies de plantas acuáticas comunes en la laguna destacan Pistia stratiotes, Begonia caroliniifolia, Lemma minor, Digitaria horizontalis, Acrostichum danaefolium, Eichhornia crassipes, Typha latifolia. La vegetación terrestre está dominada por especies como Spondias mombin, Scheelea liebmani, Sabal mexicana, Tabebuia rosea, Ceiba pentandra, Pachira aquatica, Bursera simaruba, Cocos nucifera (Magaña 1988). En la laguna se han reportado al menos 21 especies de peces, 21 de aves, 7 especies de reptiles y 10 especies de mamíferos (Gómez-González 1995).

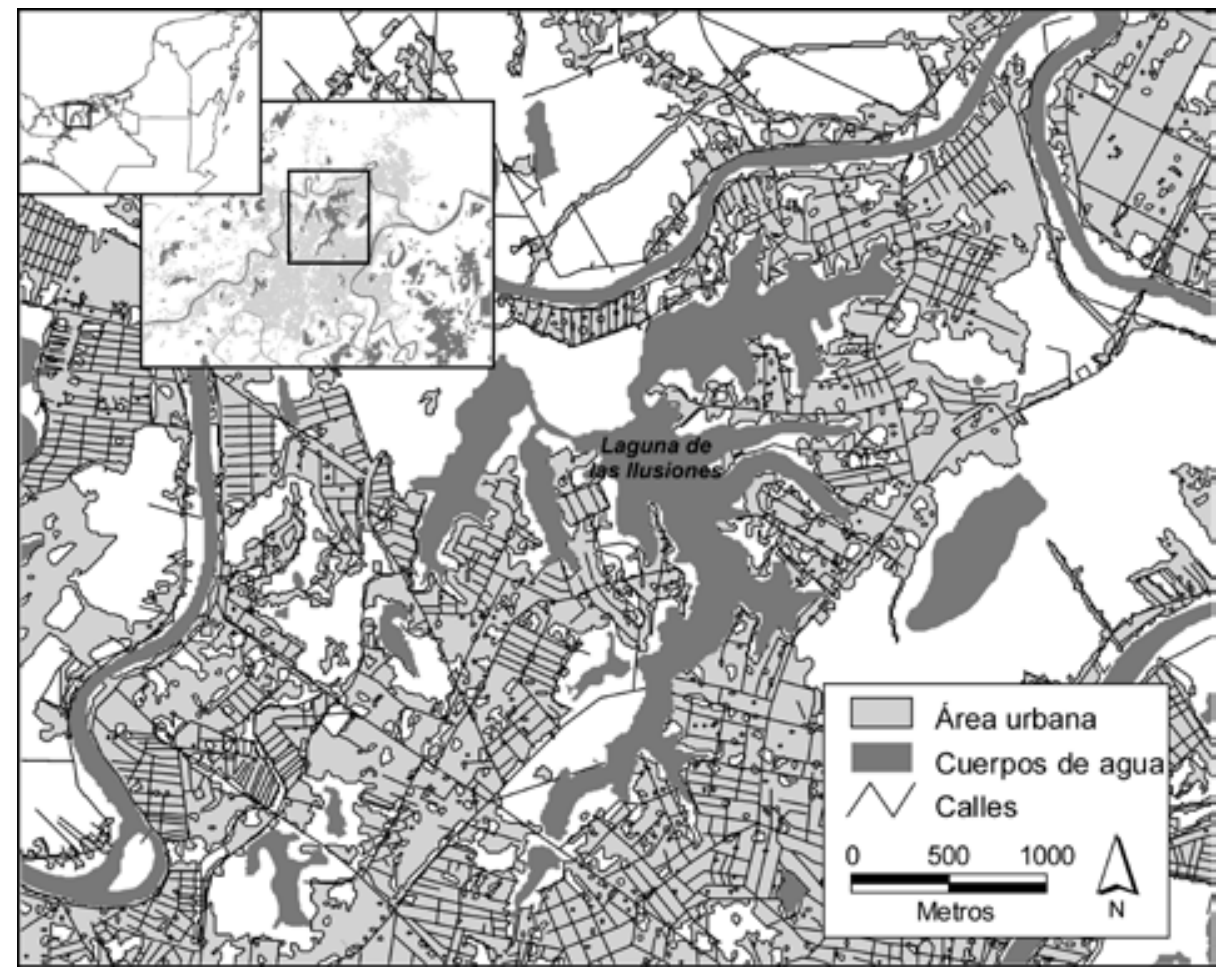

Figura 1. Localización de la Laguna de las Ilusiones en la Ciudad de Villahermosa, Tabasco, México. 
Durante la temporada de anidación del cocodrilo de pantano (mayo-agosto) en los años de 2007 y 2008, se realizaron búsquedas diurnas en dos a tres visitas por semana en la Laguna de las Ilusiones. Cada visita se inició con un recorrido por agua utilizando una lancha de aluminio de $4 \mathrm{~m}$ de largo con motor fuera de borda de $8 \mathrm{HP}$, a una velocidad no mayor a $15 \mathrm{~km} / \mathrm{h}$ y a una distancia de entre 5 y $10 \mathrm{~m}$ de la orilla. Cuando las condiciones no permitieron el uso de motor se utilizaron remos. Cada uno de los recorridos abarcó solo un sector de la laguna. El perímetro total de la laguna se recorrió en cinco visitas (10 días aproximadamente) haciendo énfasis en aquellas zonas en donde hubo rastros visibles de la presencia de cocodrilos (i.e. resbaladeros, asoleaderos y posibles veredas). Una vez localizadas las áreas potenciales, se realizaron recorridos a pie, buscando otros rastros que indicaran la presencia de nidos, que incluían senderos, montículos falsos y/o huellas a lo largo de la orilla de la laguna. El tiempo de búsqueda y encuentro del nido dependió del terreno en cada sitio. El trabajo con los nidos incluyó dos etapas separadas en el tiempo: 1) la localización y descripción de los nidos, y 2) la colecta de los huevos. En la primera etapa, una vez localizados los nidos se registró la fecha y la posición geográfica de cada uno con un receptor GPS. Así mismo, se midieron con un flexómetro $( \pm 5 \mathrm{~mm})$ la altura, el diámetro mayor y el diámetro menor de cada nido encontrado. Se midió la distancia más corta entre el nido y la orilla del agua, y se registró y clasificó el material de construcción del nido (partes vegetales, tierra y humus, materiales de desecho de uso humano). En esta etapa cada nido se abrió parcialmente mostrando una porción de la cámara de anidación para identificar el tamaño de la banda de los huevos, con esta información obteníamos la fecha aproximada de la puesta (Ferguson 1985). En la segunda etapa, cada uno de los nidos visitados fue removido y transportado en cajas de plástico individuales con medidas de $25 \times 18 \times 12 \mathrm{~cm}$ con una cama compuesta del sustrato del propio montículo y llevado al laboratorio de la Unidad de Manejo de Cocodrilos de la División académica de Ciencias Biológicas de la Universidad Juárez Autónoma de Tabasco. Sólo se transportaron los huevos intactos, aquellos parcial o totalmente quebrados, o en descomposición se contaron como parte del número total de huevos del nido, pero no se recolectaron. Cuando se observaron cambios en los montículos, como la destrucción por depredación o evidencia de otros disturbios, se registró el evento. Los nidos fueron colectados de dos a 15 días antes de la eclosión, considerando la fecha de ovoposición estimada, la fecha de colecta y remoción de los huevos fue determinada porque los nacimientos en los cocodrilianos se han reportado aproximadamente de 75 a 90 días después de la puesta (Casas-Andreu \& Rogel-Bahena 1986). Las cajas con los huevos obtenidos fueron colocadas por separado en un cuarto a temperatura ambiente (variable entre $30^{\circ}$ y $34^{\circ} \mathrm{C}$ ), y el sustrato se mantuvo húmedo rociando periódicamente con agua potable utilizando un aspersor hasta la eclosión. Los huevos fueron medidos con un calibrador vernier $( \pm 0.02 \mathrm{~mm})$ y pesados con una balanza digital Ohaus $(600 \pm 0.1 \mathrm{~g})$. Para comparar el tamaño de los huevos entre los 
nidos, se utilizó un ANOVA usando una medida de volumen de cilindro basado en el largo y ancho del huevo ( $\pm 1 \mathrm{DE})$. Se eliminaron del análisis los huevos de los nidos que eclosionaron antes de recogerlos y los que fueron destruidos por depredación o por aplastamiento. Se calculó el tamaño medio de cada hembra nidificante, a partir del ancho de los huevos de cada nido, despejando la ecuación $y=0.12 x+29.6$ (Platt et al. 2008), donde $y=$ ancho del huevo y $x=$ longitud hocico-cloaca de la hembra (LHC). Todas las crías eclosionadas que fueron obtenidas de la colecta de huevos se marcaron individualmente y se mantuvieron en un acuaterrario de $3 \times 3 \mathrm{~m}$ entre tres a cuatro semanas posteriores a su nacimiento para poder identificar el sexo, mediante la eversión y morfología del pene en los machos (Allstead \& Lang 1995). Una vez tomados los datos, las crías fueron devueltas a la laguna. Por otra parte, se obtuvieron datos adicionales similares a los tomados en este estudio de fuentes bibliográficas (Gómez 1991, Pacheco 1994) sobre la anidación de los cocodrilos de la Laguna de las Ilusiones. Los nidos de 1991-1994 se compararon con los nidos de 2007-2008 utilizando una prueba $t$ de student analizando las mismas variables descriptivas del nido para identificar los cambios en la fenología de anidación del cocodrilo de pantano en la Laguna de las Ilusiones en estos dos periodos de tiempo.

\section{RESULTADOS}

Durante el periodo de estudio (mayo-julio 2007 y mayo-julio 2008) se localizaron 22 nidos de cocodrilo de pantano. En ambos años los nidos se encontraron desde finales de mayo hasta principios de julio. El mayor número de nidos (54.5\%) se encontró entre la segunda y tercera semana de junio en ambos años. Uno de los nidos contenía 60 huevos lo cual es inusual comparado con el tamaño promedio de puestas reportado para esta especie (Casas-Andreu \& Rogel-Bahena 1986), así mismo fue notable la diferencia de tamaño entre algunos de ellos, por lo que se supuso que los huevos eran de dos puestas distintas. Para confirmar esta observación analizamos los valores de volumen de cilindro obtenidos del largo y ancho de cada uno de los huevos de este montículo. Utilizando el programa Fuzzy set Clustering tool V2 ${ }^{\circledR}$, que separa conjuntos de datos difusos de varias muestras (Equihua 1991) y los datos se dividieron claramente en dos conjuntos. Consideramos entonces 23 puestas de cocodrilo de pantano en la periferia de la Laguna de las Ilusiones. De estos 23, un nido fue depredado, dos nidos fueron destruidos por aplastamiento y un nido eclosionó antes de recolectar los huevos.

Las características generales de los montículos se compararon con otros 18 nidos reportados en 1991 y 4 nidos en 1994 (Gómez 1991, Pacheco de la Cruz 1994) (Cuadro 1). Se observó que los nidos en 2007-2008 fueron de mayor diámetro y se encontraron a mayor distancia de la orilla $(p<0.05)$. El tamaño de nidada fue mayor en 2007-2008 $(p<0.05)$. El comportamiento de resguardo de nido por parte de la hembra fue registrado en 1991, donde tres hembras daban protección total al nido 
Cuadro 1. Comparación de las características físicas de los montículos de los nidos de cocodrilo de pantano estudiados en la Laguna de las Ilusiones, Villahermosa, Tabasco durante 1991-1994 y 2007-2008. La comparación se realizó con una prueba de $t$.

\begin{tabular}{lccc}
\hline \multicolumn{1}{c}{ Características del nido } & $\begin{array}{c}\text { 1991-1994 } \\
\text { Gómez-González 1995 }\end{array}$ & $\begin{array}{c}\text { 2007-2008 } \\
\text { Este estudio }\end{array}$ & $p$ \\
\hline Diámetro mayor $(\mathrm{cm})$ & $148.85 \pm 22.4(21)$ & $179.5 \pm 56.1(22)$ & $<0.05$ \\
Diámetro menor $(\mathrm{cm})$ & $127.14 \pm 24.7(21)$ & $158.91 \pm 47.12(22)$ & $<0.05$ \\
Altura $(\mathrm{cm})$ & $50.9 \pm 20.2(21)$ & $46.32 \pm 15.2(22)$ & $>0.05$ \\
Distancia a la orilla (m) & $6.01 \pm 5.1(21)$ & $9.96 \pm 6.8(22)$ & $<0.05$ \\
Número de huevos por nido & $23.3 \pm 4.2(14)$ & $32.4 \pm 10.7(19)$ & $<0.01$ \\
\hline
\end{tabular}

saliendo del agua y permaneciendo arriba del montículo. Durante el periodo de muestreo sólo una hembra protegió el nido en ambos años y en el mismo sitio.

La composición de los materiales con que fueron construidos los nidos fue variable, en general el 53\% estuvieron construidos con vegetación compuesta principalmente de hojas, tallos y ramas delgadas, el $16 \%$ con humus y arcilla, el $8 \%$ con plantas y desechos producidos por los humanos, y el 23\% con una combinación de todos estos materiales (Cuadro 2). El tamaño promedio de nidada fue de $30.4 \pm 10.8$ huevos por nido (nidos $n=19$, huevos $n=578$, huevos $\times$ nido $\min =14$, $\max =44$ ). Existen diferencias significativas entre los nidos analizados, comparando el volumen de los huevos entre los 19 nidos ( $F=135.36, p<0.001$ nidos $n=19$, huevos $n=539$ ) (Figura 2).

En cuanto a la eclosión, 9 nidos $(N=13)$ eclosionaron en $2007(69.2 \%)$ y 8 nidos $(N=10)$ en $2008(80 \%)$. El porcentaje de nacimientos también varió entre años, del $39.8 \%$ en 2007 al 25.1\% en 2008. Para ambos años nacieron un total de 196 (36.4\%) crías. El promedio de eclosión por nido fue de $33.9 \pm 31.21$ crías por nido. Se encontraron porcentajes de eclosión por nido desde $0 \%$ hasta el $92.9 \%$. Todos los nidos fueron recolectados durante la última o las últimas dos semanas de su incubación natural, por lo que consideramos el sexo ya estaba determinado, y por eversión del pene de los individuos revisados, obtuvimos que el 100\% de los neonatos en $2007 \mathrm{y}$ 2008 fueron machos.

El tamaño de la hembras que anidaron estimado mediante la fórmula propuesta por Platt et al. (2008) mostró que la mayor parte de las 19 hembras tuvo un tamaño estimado mayor a $70 \mathrm{~cm}$ de LHC y muy pocas por debajo de esta talla (Figura 3 ).

\section{DISCUSIÓN}

Los parámetros y eventos de la anidación del cocodrilo de pantano (tamaños del nido, número de huevos, tamaño de la madre, fechas de puesta) en la Laguna de las Ilusiones coinciden de manera general con la información anteriormente publicada para la especie en otras localidades en donde se distribuye (Casas-Andreu \& Rogel Bahena 
Cuadro 2. Materiales de construcción de los nidos de cocodrilo de pantano usados en la Laguna de las Ilusiones, Villahermosa, Tabasco.

\begin{tabular}{|c|c|}
\hline \multicolumn{2}{|l|}{ Plantas } \\
\hline \multicolumn{2}{|l|}{ Pastos } \\
\hline \multirow[t]{6}{*}{ Poaceae } & Paspalum fasciculatum \\
\hline & Paspalum virgatum \\
\hline & Panicum maximum \\
\hline & Echinochloa purpurem \\
\hline & Penisetum purpurem \\
\hline & Leelsia $s p$ \\
\hline \multicolumn{2}{|l|}{ Arbustos } \\
\hline Apiaceae & Hidrocotile umbellata \\
\hline Bignoniaceae & Cydistia heterophyla \\
\hline \multirow[t]{2}{*}{ Leguminosae } & Mimosa pigra \\
\hline & Mimosa albida \\
\hline Typhaceae & Typha latifolia \\
\hline Pteridaceae & Acrostichum aureum \\
\hline Polypodeaceae & Nephrodium unitum \\
\hline Pontederiaceae & Eichornia crassipes \\
\hline \multicolumn{2}{|l|}{ Árboles } \\
\hline \multirow[t]{2}{*}{ Leguminosae } & Andira galeottiana \\
\hline & Galliandra haustoniana \\
\hline Bombacaceae & Pachira acuática \\
\hline \multirow[t]{2}{*}{ Arecaceae } & Cocos nucifera \\
\hline & Sabal sp \\
\hline \multicolumn{2}{|l|}{ Material inorgánico } \\
\hline \multicolumn{2}{|l|}{ Tierra y arcilla } \\
\hline Desechos plásticos & $\begin{array}{l}\text { Bolsas y botellas de diferentes tamaños, vasos, platos y cubiertos } \\
\text { desechables, empaques de dulces y medicamentos, partes de } \\
\text { electrodomésticos, neumáticos. }\end{array}$ \\
\hline Otros desechos & $\begin{array}{l}\text { Latas de comida y pintura, restos de zapatos, cuerdas, mallas de metal, } \\
\text { botellas de vidrio, pañales y toallas desechables, cajas de cartón. }\end{array}$ \\
\hline
\end{tabular}

1986, Platt et al. 2008). Sin embargo, algunas otras características difieren, como materiales de anidación no típicos, proporción sexual sesgada hacia los machos, bajo éxito de eclosión comparado con otras especies de cocodrilianos en vida silvestre, así como una diferencia notable en el tamaño de puesta entre 1991 y 2007-2008 en el mismo lago.

Nuestros resultados indican que al menos existen 13 hembras con capacidad de reproducción en la laguna, aunque el número de nidos encontrados en 2008 fue menor $(N=10)$. Observaciones y conteos prospectivos en el área, indican un numero importarte de cocodrilos (199 individuos en promedio por observación) (López-Luna, datos sin publicar), mucho mayor si se compara con trabajos demográficos previos (Pacheco de la Cruz 1996 reporta 62 individuos y Gómez-Rodríguez 2003 reporta 96 indi- 


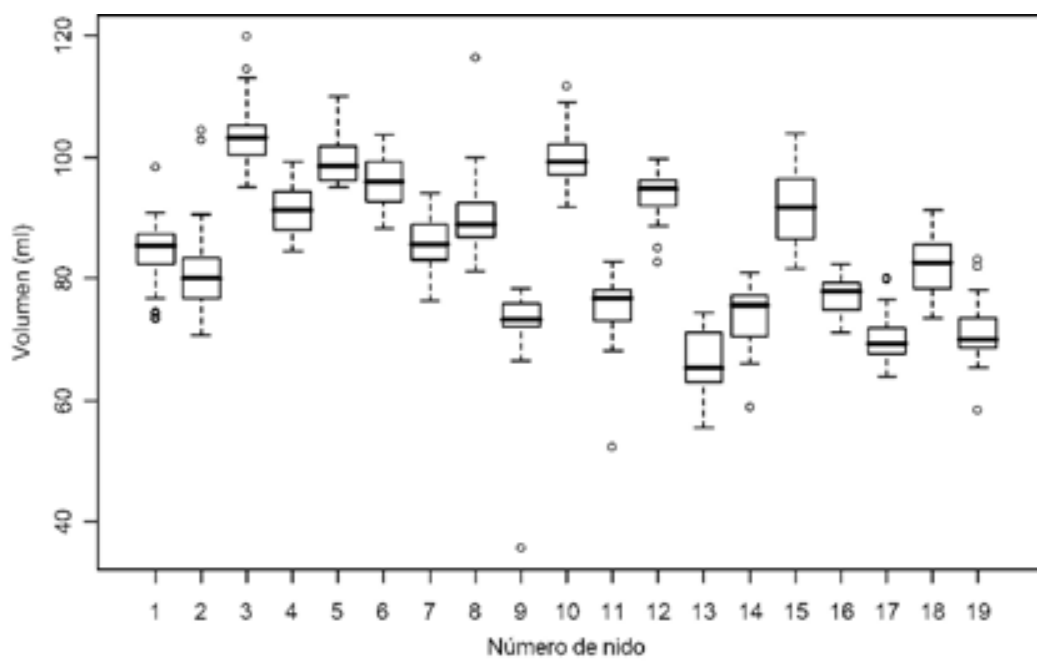

Figura 2. Promedio, desviación estándar y cuartiles del volumen de los huevos por nido de cocodrilo de pantano en la Laguna de las Ilusiones, en los periodos de reproducción de 2007-2008.

viduos). Sin embargo, el número de hembras reproductoras parece haber disminuido pues en 1991 se registraron 18 nidos en la laguna utilizando un esfuerzo de muestreo similar (Gómez-González 1995) al de este estudio. Una de las razones podría ser que las hembras están construyendo los nidos mucho más alejados de la orilla, en sitios poco accesibles y difíciles de encontrar. Otra posible causa es que el reclutamiento de hembras reproductoras en la Laguna de las Ilusiones sea menor que el de los machos, lo que se explicaría también con el amplio sesgo de machos naciendo por nido, de tal forma que si esta tendencia viene de años anteriores, significaría que pocas hembras han estado naciendo en años previos al 2007.

Actualmente los nidos tienen un mayor diámetro en la base del nido (área basal) que en 1991-1994 (Gómez-González 1995, Pacheco de la Cruz 1996). Esto puede deberse a que la expansión de la ciudad de Villahermosa (3.2\% anual en población INEGI 2005) ha provocado cambios importantes en los hábitat riparios que rodean la laguna, estos cambios podrían alterar desde la disponibilidad del material de construcción hasta el microclima de los nidos, en este último caso es posible que la hembra se vea obligada a modificar el tamaño de su nido para mantener condiciones óptimas de temperatura y humedad para el desarrollo de los huevos. En ese contexto, se ha documentado que comportamientos como orinar o salpicar el nido durante su construcción o remodelación sugieren que se debe a iniciar la descomposición de materia vegetal (Ferguson, 1985) o regular la humedad (McIlhenny, 1934). Se conoce que ciertas especies de cocodrilianos remodelan el nido durante la incubación además de 


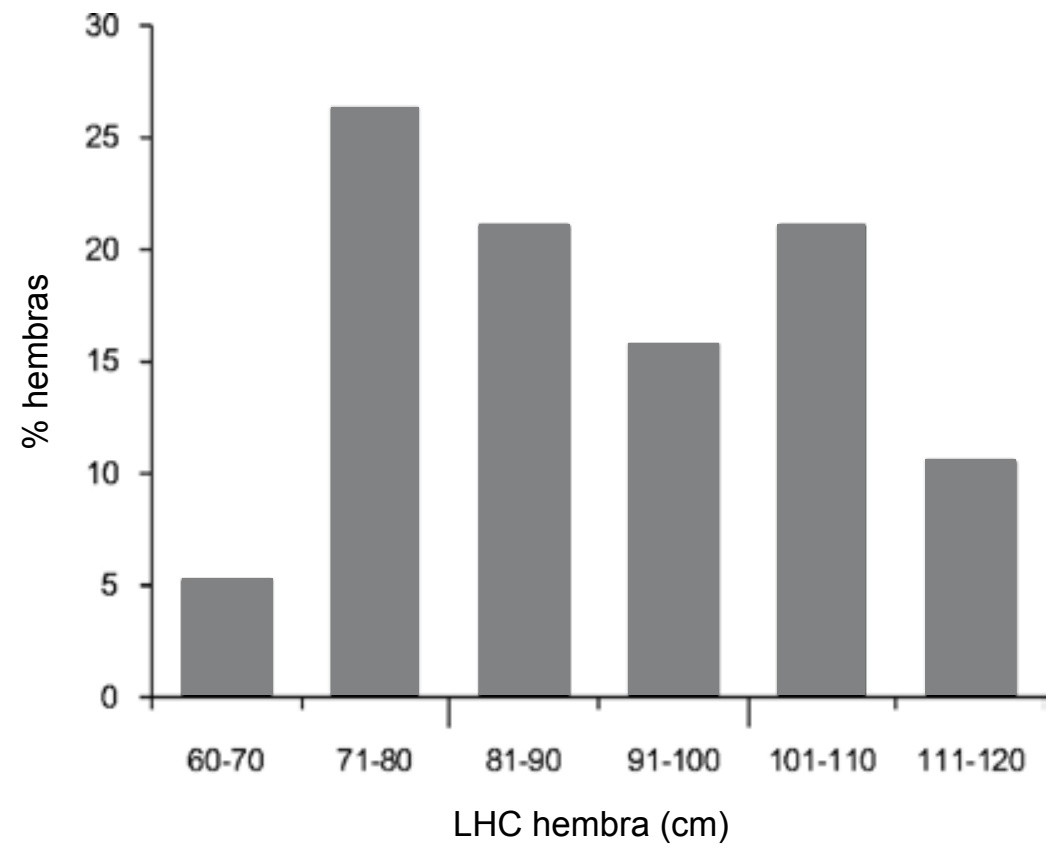

Figura 3. Distribución del tamaño estimado para las hembras reproductoras de cocodrilo de pantano (Longitud Hocico-Cloaca; LHC) en la Laguna de las Ilusiones. La estimación del tamaño se obtuvo a partir del ancho promedio de los huevos de cada nido utilizando la ecuación $y=0.12 x+29.6$ (Platt et al. 2008), donde $y=$ ancho del huevo y $x=$ LHC.

salpicar u orinar sobre el nido (Álvarez del Toro 1974, Deitz \& Hines 1980, Ferguson 1985, Lang, 1987, McIlhenny 1934, Venegas de Anaya, 2000) por lo que sería factible pensar que controlen la cantidad de material de construcción del nido si eso tiene un efecto en el aumento o disminución de la temperatura de incubación. Se ha sugerido también que la hembra podría monitorear la temperatura y/o humedad del nido con los receptores sensoriales localizados en sus mandíbulas (Seebacher \& Franklin 2005, Soares 2002) y modificar el nido en consecuencia. Además, las condiciones ambientales como la cantidad de lluvia y el hábitat están relacionados estrechamente con la dinámica reproductora de los cocodrilianos (Campos \& Magnusson 1995).

Así mismo, las modificaciones en los hábitat riparios puede cambiar muchos aspectos de la vida silvestre, como la distribución y abundancia de las comunidades animales (Kelsey \& West 2001), y afectan la selección de los sitios de anidación de algunos reptiles acuáticos como tortugas dulceacuícolas (Kolbe \& Janzen 2001). Es así que la búsqueda de materiales de construcción para el nido por parte de la hembra puede ser causante de las características del nido. En relación a esto último, podemos observar que los nidos en 2007-2008 se encontraron más lejos de la orilla y menos 
protegidos por las hembras comparado con la condición observada en 1991-1994 (Gómez-González 1995, Pacheco de la Cruz 1996). El crecimiento de la ciudad ha provocado que la orilla de la laguna presente una gran alteración y presencia humana elevando y compactando el suelo. Esto puede resultar en que las hembras se alejen de la orilla, buscando terrenos adecuados para anidar, que incluyan mejores materiales de construcción, humedad, sombra o luz disponible, entre otros factores físicos, o bien que den mayor seguridad, tanto al nido como a la madre nidificante. En otras especies de reptiles acuáticos, como algunas tortugas dulceacuícolas, la selección del sitio de puesta se relaciona con el incremento del éxito de supervivencia de las crías, pero también en función de que la hembra encuentre el menor riesgo al anidar (Spencer 2002). Es posible que las hembras de cocodrilo de pantano en la Laguna de las Ilusiones estén siguiendo una estrategia similar, es decir, a pesar de que alejarse de la orilla puede poner en riesgo el éxito de la nidada al ofrecer menos protección al nido, la hembra puede sentirse más segura y menos molestada. Se ha observado que nidos muy alejados de la orilla son muy propensos a la depredación, ya que la hembra no tiene oportunidad de proteger al nido (Kofron 1989). Sin embargo, solo se registró un nido con señales de depredación en el periodo de muestreo. Los depredadores potenciales observados en el área son perros (Canis familiaris), mapaches (Procyon lotor) coatís (Nasua narica) y tlacuaches (Didelphis sp.). Los recursos alimenticios (provenientes principalmente de restos de comida humana, basura y desechos orgánicos) que ofrece la ciudad a depredadores oportunistas (Gehrt 2004) como los observados en Villahermosa, podrían reducir el riesgo de depredación hacia los nidos de cocodrilo de pantano con poca protección, favoreciendo el establecimiento de nidos lejanos a la orilla donde la hembra tiene poca oportunidad de defenderlo.

En los estudios anteriores (Gómez-González 1995, Pacheco de la Cruz 1996) no se reporta la construcción de nidos con basura producida por el humano. En este estudio este componente resultó muy importante en la construcción de los nidos ya que el $31 \%$ de los nidos tuvo un porcentaje variable de basura. Esto es importante porque dos nidos fueron aplastados posiblemente por personas que confundieron los nidos con acumulaciones de basura. Uno de estos nidos estaba construido sobre vegetación flotante semejante a lo descrito por Pérez-Higareda (1980) y se perdió por hundimiento debido a la acumulación de desechos de jardinería urbana. Este factor de mortalidad puede ser muy impactante ya que las márgenes de la Laguna de las ilusiones se rozan, tumban y acumulan materia orgánica de manera regular, pudiendo aplastar a los nidos en el área. Aunque no hay datos que indiquen que la hembra de cocodrilo puede aplastar fatalmente a sus huevos poniéndose encima del nido al darle mantenimiento o defendiéndolo, la actividad de limpieza y acumulación de basura sobre los nidos por seres humanos, podría ser fatal para los huevos, como se observo en esos dos nidos encontrados.

La relación entre el tamaño de la nidada y el tamaño de la hembra en cocodrilos y en otros reptiles ha sido un tema de controversia. En algunos estudios con coco- 
drilos no se ha observado una relación significativa entre estas dos variables (Joanen 1969, Casas-Andreu \& Rogel-Bahena 1986). En contraste, otros trabajos sugieren que hembras más grandes tienden a producir nidadas más chicas (Thorbjarnarson \& Hernández 1993, Larriera et al. 2004), y otros estudios sugieren que posiblemente a mayor tamaño de la hembra las variables como el tamaño de la nidada, el ancho de los huevos y el tamaño de los neonatos también serían mayores. (Montini et al. 2006).

El número de huevos en la Laguna de las Ilusiones se ha visto incrementado recientemente en comparación con los nidos contados en 1991 en el mismo lugar (Gómez-González 1995). Una de las causas probables es un potencial cambio del tamaño de las hembras a lo largo del tiempo, influyendo en las diferentes características del nido. En Belice la distribución de tallas en hembras que anidan presenta pocos individuos tanto pequeños ( $<80 \mathrm{~cm}$ LHC) como muy grandes $(>100 \mathrm{~cm}$ LHC) y la mayoría en las tallas intermedias (Platt et al. 2008). En nuestro estudio la distribución difiere, ya que se observó un grupo importante de hembras maduras jóvenes (de 60 a $80 \mathrm{~cm}$ de LHC) y un gran número de hembras maduras que van de grandes (de 81 a $100 \mathrm{~cm}$ de LHC) a muy grandes (de $101 \mathrm{a} 120 \mathrm{~cm}$ de LHC), sin que las tallas intermedias sobresalgan. Esta distribución sugiere que las hembras permanecen por largo tiempo en el área, y el reclutamiento de nuevas hembras en la población puede estar restringido por el territorio de anidación, y como se explicó anteriormente por el nacimiento de una gran proporción de machos en los últimos años, reclutando a la larga más machos que hembras reproductoras.

El éxito de eclosión fue menos de $35 \%$ de los huevos puestos en promedio durante el año eclosionan, lo que sería un porcentaje reducido si lo comparamos con estudios de otras especies en vida silvestre (gaviales y C. acutus) donde el éxito de eclosión va del 60 al 88\% (Hussain 1999, Cupul-Magaña et al. 2004). No existen datos publicados sobre el éxito de eclosión del cocodrilo de pantano en vida silvestre, sin embargo, estudios en cautiverio de esta especie mencionan un promedio de eclosión del 40.7\% (Casas Andreu et al. en prensa).

Todos los nacimientos de cocodrilo de pantano registrados en 2007-2008 fueron machos. Se reconoce el efecto de la temperatura en la determinación sexual (TDS) en cocodrilianos (Lang \& Andrews 1994, Deeming 2004) y el cocodrilo de pantano no es la excepción. Sin embargo, los estudios de TDS con la especie son contradictorios con respecto al modelo de TDS y las temperaturas umbrales (Aguilar-Miguel 1994, Lang \& Andrews 1994). El ambiente de la ciudad es importante en estos aspectos, el efecto de "isla urbana de calor" promueve un aumento y mantenimiento alto en la temperatura de las ciudades (Voogt 2002), que podría influir en la temperatura de incubación y favorecer la formación de machos en el cocodrilo de pantano. Así mismo, se han encontrado contaminantes en el ambiente que pueden actuar como disruptores hormonales (ver revisión de Salame-Méndez et al. 2008) que pueden sesgar la proporción sexual en el desarrollo embrionario de cocodrilianos (Stoker et al. 2003, 
2008). Por consiguiente, la sinergia de la "Isla Urbana de Calor" y los disruptores hormonales presentes en la Laguna de las Ilusiones puede tener un amplio efecto en la determinación de los sexos en la población de cocodrilo de pantano. Por otro lado, se han detectado alteraciones en el material génico de la población de cocodrilo de pantano en la Laguna de las Ilusiones (Aguirre-Álvarez 2009), y se ha demostrado que pesticidas pueden tener efectos muy importantes en el desarrollo de los individuos (Polleta et al. 2009). Desconocemos también el grado de endogamia existente en los cocodrilos de la Laguna de las Ilusiones, la cual tiene poca o nula conexión con otros cuerpos de agua. Es posible que estos factores genéticos, pueden favorecer de alguna manera la formación de machos en el área de estudio.

Los cocodrilos que habitan la Laguna de las Ilusiones son parte del paisaje urbano, se reproducen de manera regular y han estado utilizando los recursos que ofrece una ciudad. Los depredadores potenciales también tienen recursos alimentarios suplementarios que coadyuvan a que la mayoría de los nidos llegue hasta la temporada de eclosión sin ser depredados, sin embargo, es el mismo entorno urbano el que podría causar la baja tasa de eclosión y el sesgo hacia los machos debido a temperaturas de incubación extremas. De seguir con esta tendencia a largo plazo existirá poco reclutamiento de hembras a la población, y las hembras disponibles se harán más viejas, reduciendo la calidad genética y el potencial reproductor (Lance 2003), aunado a una alta tasa de reclutamiento de machos, que llevará a una población monosexual no reproductora. El problema será magnificado debido a las bajas probabilidades de inmigración por aislamiento de la propia laguna. Ante estos escenarios se hace necesario un manejo de la población de cocodrilos en la laguna de las Ilusiones, que pueden ir desde la reintroducción de hembras, hasta el manejo de los nidos en sistemas de incubación artificial que promuevan el desarrollo de hembras, además de realizarse más investigación en torno a los factores que promueven el sesgo hacia los machos en esta población urbana de cocodrilos de pantano.

AGRADECIMIENTOS. A la Universidad Juárez Autónoma de Tabasco, particularmente al Dr. Wilfrido M. Contreras Sánchez de la División Académica de Ciencias Biológicas por el apoyo académico a este trabajo. A la Secretaria de Recursos Naturales y Protección al Ambiente del Gobierno Estado de Tabasco por las facilidades otorgadas, así como el uso de lanchas y motor cuando fue necesario. Al Scott Neotropical Fund de la Cleveland Zoological Society y Cleveland Metroparks Zoo por el financiamiento parcial para el desarrollo de este trabajo. Este trabajo se realizó con la aprobación de la Secretaria de Medio Ambiente y Recursos Naturales con el permiso Num. SGPA/DGVS/07231. De manera especial a los estudiantes y pasantes de la licenciatura en Biología Carlos Vázquez-Hermosillo, Mariana del C. González-Ramón, Gustavo Rodríguez-Azcuaga, Ezri Rodríguez-Villarreal, Osiris Escalante-Aguilar y Filiberto De la Cruz-Sánchez y a los Biólogos Roberto (Toby) Ramos Targarona, José Antonio Germán Arellano y al por el apoyo brindado. A la Dra. Eunice Pérez-Sánchez, M en C Arlette Hernández-Franyutti, Dr. León David Olivera-Gómez y a la M en C Claudia Elena Zenteno Ruíz por sus sugerencias al manuscrito. 
López-Luna et al.: Nidos de Crocodylus moreletti en un paisaje urbanizado

\section{LITERATURA CITADA}

Aguilar-Miguel, X. 1994. Efecto de la temperatura de incubación sobre la determinación del sexo en Crocodylus acutus y C. moreletii. Tesis de Maestría. Universidad Nacional Autónoma de México, México, D. F.

Aguirre-Álvarez, I. K. 2009. Estudio de Genotoxicidad en una población de cocodrilos de pantano (Crocodylus moreletii) de la Laguna de las Ilusiones, mediante la técnica de electróforesis unicelular en gel. Tesis de Licenciatura. División Académica de Ciencias Biológicas. Universidad Juárez Autónoma de Tabasco, Villahermosa, Tabasco, México.

Allsteadt, J. \& J. W. Lang. 1995. Sexual dimorphism in the genital morphology of young American alligators, Alligator mississippiensis. Herpetologica, 51: 314-325.

Bodie, J. R. 2001. Stream and riparian management for freshwater turtles. Journal of Environmental Management, 62: 443-455.

Campbell, H. W. 1972. Ecological or phylogenetic interpretations of crocodilian nesting habits. Nature, 238: 404-405.

Campos, Z. \& W. Magnusson. 1995. Relationships between rainfall, resting habitat and fecundity of Caiman crocodilus yacare in the Pantanal, Brazil. Journal of Tropical Ecology, 11: 351-358.

Casas-Andreu, G., G.Barrios-Quiroz \& R. Macip-Ríos. 2011. Reproducción en Cautiverio de Crocodylus moreletii en Tabasco, México. Revista Mexicana de Biodiversidad, 82(1): 1-18.

Casas-Andreu, G. \& M. Guzmán-Arroyo. 1970. Estado actual de las investigaciones sobre cocodrilos mexicanos. Secretaría de Industria y Comercio. Instituto Nacional de Investigaciones Biológico Pesqueras. Serie Divulgación, 3: 1-52.

Casas-Andreu, G. \& A. Rogel-Bahena. 1986. Observaciones sobre los nidos y las nidadas de Crocodylus moreletii en México. Anales de. Instituto de Ciencias del Mar y Limnoogia Universidad Nacional Autónoma de México, 12: 232-330.

Cupul-Magaña, F. G., A. De Niz-Villaseñor, A. Reyes-Juárez \& A. Rubio-Delgado. 2004. Historia natural del cocodrilo americano (Crocodylus acutus) en el estero Boca Negra, Jalisco, México: anidación y crecimiento de neonatos. Ciencia y Mar, 16: 31-42.

Deeming, D. C. 2004. Prevalence of TSD in Crocodilians. Pp. 33-41. In: Valenzuela, N. \& V. Lance (Eds.). Temperature-dependent sex determination in vertebrates. Smithsonian Books, Washington.

Domínguez-Laso, J. 2006. Determinación del estado de las poblaciones silvestres del cocodrilo de pantano (Crocodylus moreletii) en México y evaluación de su estatus en la CITES. Instituto de Historia Natural y Ecología. Informe final SNIB-CONABIO proyecto No. CS009. México D. F.

Equihua, M. 1991. Fuzzy clustering of ecological data. Journal of Ecology, 78: 519-534.

Faeth, S. H., P. S. Warren, E. Shochat \& W. A. Marussich. 2005. Trophic dynamics in urban communities. Bioscience, 55: 399.

García, E. 1973. Modificaciones al sistema de clasificación climática de Koeppen (Adaptada para la República Mexicana). Instituto de Geografía, Universidad Nacional Autónoma de México. México D. F.

Gehrt, S. D. 2004. Ecology and Management of Striped Skunks, Raccoons, and Coyotes in Urban Landscapes. Pp. 81-104. In: N. Fascione, A. Delach, \& M. Smith (Eds). People and Predators: From Conflict to Coexistence. Island Press. Washington.

Gómez-González, F. 1995. Descripción de la nidación de Crocodylus moreletii (Dumeril y Dumeril, 1851) en la Laguna de las Ilusiones, Municipio del Centro, Tabasco, México. Tesis de Licenciatura, Facultad de Biología, Universidad Veracruzana.

Gómez-Rodríguez, W. 2003. Estudio preliminar para estimar la densidad relativa y distribución de Crocodylus moreletii (Cocodrilo de Pantano) en la Laguna de las Ilusiones en el período Julio- diciembre/2002, Municipio de Centro, Tabasco. Tesis de Licenciatura en Ecología, División. Académica de Ciencias Biológicas Universidad Juárez Autónoma de Tabasco. 
Grimm, N. B., S. H. Faeth., N. E.Golubiewski, C. L. Redman, J. Wu, X. Bai \& J. M. Briggs. 2008. Global change and the ecology of cities. Science, 319(5864): 756-60.

Hunt, R. H. 1973. Breeding Morelet's crocodile Crocodylus moreletii at Atlanta Zoo. International Zoo YearBook, 13: 103-105.

Hunt, R. H. 1975. Maternal behaviour in the Morelet's crocodile Crocodylus moreletii. Copeia, 1975: 763-764.

Hunt, R. H. 1977. Aggressive behavior by adult Morelet's crocodiles Crocodylus moreletii toward young. Herpetologica, 33: 195-201.

Hunt, R. H. 1987. The Morelet's crocodile project of Zoo Atlanta pp 158-161. In: Annual Conference Proceedings American Association of Zoological Parks and Aquariums. Wheeling WV.

Hussain S. A. 1999. Reproductive success, hatchling survival and rate of increase of gharial Gavialis gangeticus in National Chambal Sanctuary, India. Biological Conservation, 87: 261-268.

Instituto Nacional de Ecología (INE). 1999. Proyecto para la Conservación, Manejo y Aprovechamiento Sustentable de los Crocodylia en México. SEMARNAP, México.

Joanen, T. 1969. Nesting ecology of alligators in Louisiana. Proceedings of the Annual Conference of Southeastern Association of Game and Fish Commissioners, pp. 141-151.

Joyal, L. A., M. McCollough \& M. L. Hunter Jr. 2001. Landscape ecology approaches to wetland species conservation: a case study of two turtle species in southern Maine. Conservation Biology, 15: $1755-1762$.

Kelsey, K. A. \& S. D. West. 2001. Riparian wildlife. Pp. 235-258. In: Naiman, R. J., Bilby, R. E. (Eds.) River Ecology and Management. Springer, New York.

Kofron, C. P. 1989. Nesting ecology of the Nile crocodile (Crocodylus niloticus). African Journal of Ecology, 27: 335-341.

Lang, J. W. \& H. V. Andrews. 1994. Temperature-dependent sex determination in Crocodilians. The Journal o Experimental Zoology, 270: 28-44.

Larriera, A., C. I. Piña, P. Siroski, \& L. M. Verdade. 2004. Allometry of reproduction in wild BroadSnouted caimans (Caiman latirostris) Journal of Herpetology, 38: 301-304.

Magaña-Alejandro, M. 1988. La Vegetación de la Laguna de las Ilusiones. Universidad Juárez Autónoma de Tabasco, Villahermosa, Tabasco.

Montini, J. P., C. I. Piña, A. Larriera, P. Siroski \& L. M. Verdade. 2006. The relationship between nesting habitat and hatching success in Caiman latirostris (Crocodylia, Alligatoridae). Phyllomedusa, 5: 91-96.

Pacheco de la Cruz, C. 1996. Análisis Preliminar sobre el estado actual de la población de Crocodylus moreletii en la Laguna de Las Ilusiones, Villahermosa, Tabasco, México. Tesis Licenciatura. División Académica De Ciencias Biológicas. Universidad Juárez Autónoma de Tabasco.

Pereyra-Díaz, D., U. Bando-Murrieta \& M. A. Natividad-Baizabal. 2004. Influencia de La Niña y El Niño sobre la precipitación de la Ciudad de Villahermosa, Tabasco, México. Universidad y Ciencia, 20: 33-38.

Pérez-Higareda, G. 1980. Notes on nesting of Crocodylus moreletii in southern Veracruz, Mexico. Bulletin of the Maryland Herpetological Society, 16: 52-53.

Platt, S. G. \& J. B.Thorbjarnarson. 2000. Population status and conservation of Morelet's crocodile, Crocodylus moreletii, in northern Belize. Biological Conservation, 96: 21-29.

Platt, S. G., T. R. Rainwater, J. B. Thorbjarnarson \& S. T. McCurry. 2008. Reproductive dynamics of a tropical freshwater crocodilian: Morelet's crocodile in northern Belize. Journal of Zoology, 275: 177-189.

Poletta G. L., A. Larriera, E. Kleinsorgeb \& M. D. Mudryc. 2009. Genotoxicity of the herbicide formulation Roundup ${ }^{\circledR}$ (glyphosate) in broad-snouted caiman (Caiman latirostris) evidenced by the 
López-Luna et al.: Nidos de Crocodylus moreletti en un paisaje urbanizado

Comet assay and the Micronucleus test. Mutation Research/Genetic toxicology and Environmental Mutagenesis, 672: 95-102.

Ross, J. P. (Ed.). 1998. Crocodiles. Status Survey and Conservation Action Plan. 2nd ed. Crocodile Specialist Group, SSC/IUCN The World Conservation. Consultado en línea en http://www.flmnh. ufl.edu/natsci/herpetology/act-plan/plan1998a.htm.

Ross, J. P. 2000. Crocodylus moreletii. In: IUCN 2010. IUCN Red List of Threatened Species. Version 2010.1. <www.iucnredlist.org>. Downloaded on 20 February 2010.

Salame-Méndez, A., F. Méndez-de la Cruz, G. Aguirre-León \& H. Serrano. 2008. Disrupción endocrina de la diferenciación sexual. ContactoS, 70: 43-49.

SEMARNAT. 2002. Norma Oficial Mexicana NOM-059-SEMARNAT-2001 Protección AmbientalEspecies Nativas de México de Flora y Fauna Silvestres-Categorías De Riesgo y Especificaciones para su Inclusión, Exclusión o Cambio-Lista De Especies En Riesgo. Miércoles 6 de marzo de 2002 Diario Oficial (Segunda Sección).

Shochat, E., P. S. Warren, S. H. Faeth, N. E. McIntyre \& D. Hope. 2006. From patterns to emerging processes in mechanistic urban ecology. Trends in Ecology and Evolution, 21: 186-191.

Sigler, L. \& E. Sarmiento-Marina. 2004. Highest nesting record for Morelet's Crocodile, Crocodylus moreletii, in Chiapas, Mexico. Crocodile Specialist Group Newsletter, 23: 10.

Sigler, L. \& González-Blanco, S. 1994. Reproduction of Morelet's crocodile in Mexico City. Crocodile Specialist Group Newsletter, 13: 19.

Spencer, R. C. 2002. Experimentally testing nest site selection: Fitness trade-offs and predation risk in turtles. Ecology, 83: 2136-2144.

Stoker, C., P. M. Beldoménico, V. L. Bosquiazzo, M. A. Zayas, F. Rey, H. Rodríguez, M. Muñozde-Toro \& E. H. Luque. 2008. Developmental exposure to endocrine disruptor chemicals alters follicular dynamics and steroid levels in Caiman latirostris. General and Comparative Endocrinology, 156: 603-612.

Stoker, C., F. Rey, H. Rodriguez, J. G. Ramos, P. Sirosky, A. Larriera, E. H. Luque \& M. Muñoz-de-Toro. 2003. Sex reversal effects on Caiman latirostris exposed to environmentally relevant doses of the xenoestrogen bisphenol A. General and Comparative Endocrinology, 133: 287-296.

Thorbjarnarson, J. B., X. M. Wang \& S. Ming. 2002. Wild populations of the Chinese alligator approach extinction. Biological Conservation, 103: 93-102.

Thorbjarnarson, J. B. 1996. Reproductive characteristics of the order Crocodylia. Herpetologica, 52: $8-24$.

Thorbjarnarson, J. B., \& G. Hernandez. 1993. Reproductive ecology of the Orinoco Crocodile (Crocodylus intermedius) in Venezuela. I. Nesting ecology and clutch relationships. Journal of Herpetology, 27: 363-370.

Lance, V. A. 2003. Alligator physiology and life history: the importance of temperature. Experimental Gerontology, 38: 801-805.

Verdade, L. M. 1998. Caiman latirostris. Pp. 18-20. In: J. P. Ross (Ed.). Crocodiles: status survey and conservation action plan. IUCN-The World Conservation Union, Gland, Switzerland.

Voogt, J. A. 2002. Urban Heat Island. Pp. 660-666. In: T. Munn (Ed ). Encyclopedia of Global Environmental Change, vol. 3. Wiley, Chichester. 